\title{
$\partial$-Reducing Dehn Surgeries and 1-bridge Knots
}

\author{
Ying-Qing $\mathrm{Wu}^{1}$
}

A 3-manifold is $\partial$-reducible if $\partial M$ is compressible in $M$. By definition, this means that there is a disk $D$ properly embedded in $M$ so that $\partial D$ is an essential curve in $\partial M$. The disk $D$ is called a compressing disk of $\partial M$, or a $\partial$-reducing disk of $M$.

Now suppose $M$ is a $\partial$-reducible manifold. Let $K$ be a knot in a 3-manifold $M$ such that $\partial M$ is incompressible in $M-K$. A Dehn surgery on $K$ is called $\partial$-reducing if the surgered manifold is $\partial$-reducible. It is known that in generic case there are very few $\partial$ reducing surgeries. More precisely, if there is no essential annulus in $M-\operatorname{Int} N(K)$ with one boundary component in both $\partial M$ and $\partial N(K)$, then there are at most three $\partial$-reducing surgeries [12]. Here $N(K)$ denotes a regular neighborhood of $K$ in $M$. Examples of Berge $[1,2]$ and Gabai [4] show that "three" is the best possible in general. However, all the examples in the above papers are 1-bridge knots. Intuitively, if a knot is "very knotted" in a manifold, then it should admit no $\partial$-reducing surgeries. This leads to the 1-bridge problem of Berge, which asks if 1-bridge knots are the only ones that admit nontrivial $\partial$-reducing surgeries. To be more precise, we need the following definitions.

Definition (1) A knot $K$ in $M$ is a $\partial$-reducing knot if $\partial M$ is compressible in $M$ and incompressible in $M-K$, and $K$ admits a non-trivial $\partial$-reducing surgery.

(2) A knot $K$ in $M$ is a boundary parallel (or 0-bridge) knot if it is isotopic to a simple closed curve on $\partial M$.

(3) $K$ is a 1-bridge knot if $K$ is isotopic to $\alpha \cup \beta$, where $\alpha$ is an arc on $\partial M, \beta$ is properly embedded in $M$, and there is an $\operatorname{arc} \beta^{\prime}$ on $\partial M$ such that $\beta \cup \beta^{\prime}$ bounds a disk.

(4) $K$ is a 1-tunnel knot (or generalized 1-bridge knot) if $K$ is isotopic to $\alpha \cup \beta$, where $\alpha$ lies on $\partial M$, and $\beta$ is disjoint from some compressing disk of $\partial M . \alpha \cup \beta$ is called a 1-tunnel presentation of $K$.

\footnotetext{
${ }^{0}$ Mathematics subject classification (1991): Primary 57N10, secondary 57M25.

${ }^{1}$ Partially supported by NSF grant DMS 9102633.
} 
(5) Given a $\partial$-reducing knot $K$, let $K^{\prime}$ be the central curve of the Dehn filling solid torus in the surgered manifold corresponding to a $\partial$-reducing surgery on $K$. Then $K^{\prime}$ is called a dual knot of $K$ (with respect to this $\partial$-reducing surgery.)

1-bridge (resp. 1-tunnel) Conjecture: If $K$ is a $\partial$-reducing knot in $M$. Then either $K$ or its dual $K^{\prime}$ with respect to a $\partial$-reducing surgery is a 1-bridge (resp. 1-tunnel) knot.

Note that by our definition a 0-bridge knot is a 1-bridge knot, and a 1-bridge knot is a 1-tunnel knot, but not vise versa. Clearly, the 1-bridge conjecture is stronger than the 1-tunnel conjecture. Earlier there is a problem asking if $\partial$-reducing knots are always 1-bridge knots [5]. This is true for knots in solid tori (see Section 1), but it is false if no further conditions are posted on $K$ or $M$. A simple example is given by considering a 0 -bridge knot $K$ in some $M$ such that $\partial M$ is compressible in $M$ but not in $M-K$. Surgery on $K$ along the preferred longitude will produce a $\partial$-reducible manifold, in which the dual knot $K^{\prime}$ is a $\partial$-reducing knot, but it cannot be a 1-bridge knot unless $M$ is a solid torus and $K$ is the central curve. Other simple examples are provided by surgery on $(p, 1)$ cable $K$ of a 0 -bridge knot. One may choose the slope so that the dual knot $K^{\prime}$ is a $\partial$-reducing knot, but again $K^{\prime}$ is generally not a 1-bridge knot. See Section 1 for more details. These examples show that a $\partial$-reducing knot may not be a 1-bridge knot. However they do not provide counter examples to the above conjectures. The purpose of this paper is to approach these problems from both positive and negative sides.

In section 1 we will prove the following theorem.

Theorem 1 Suppose $K$ is a $\partial$-reducing knot in a 3-manifold $M$ with $M-K$ irreducible, and suppose there is a torus $T$ which separates $K$ from $\partial M$ but does not bound a regular neighborhood of $K$. Then either $M$ is a solid torus and $K$ is a 1-bridge braid, or one of $K$ and $K^{\prime}$ is a $(p, \pm 1)$ cable $(p>1)$ of some 0 -bridge knot $L$ with respect to some preferred meridian-longitude pair. Especially, one of $K$ and $K^{\prime}$ is a 1-bridge knot.

This theorem gives some positive answer to the 1-bridge Conjecture in the case when there is a torus separating $K$ from $\partial M$. As an application, we will give all satellite $\partial$-reducing knots, as well as the $\partial$-reducing surgeries they admit.

Although the 1-bridge Conjecture is true for satellite knots, the answer in general case is negative: 
Theorem 2 There is a compact 3-manifold $M$ and a $\partial$-reducing knot $K$ in $M$, such that neither $K$ nor its dual $K^{\prime}$ with respect to the $\partial$-reducing surgery are 1-tunnel knots.

It shows that even the 1-tunnel Conjecture is not true in general, if there is no further restrictions on the knots or the manifolds.

Since all the examples of $\partial$-reducing knots in $[1,2,4]$ are knots in solid tori, it is now very natural to restrict our attention to the problem of whether the 1-bridge conjecture or 1-tunnel conjecture is true for knots in handlebodies. These problems are still open, but we will show in section 3 that the two conjectures are equivalent to each other:

Theorem 3 If $K$ is a $\partial$-reducing 1-tunnel knot in a handlebody $M$, then it is a 1-bridge knot.

Another interesting property of 1-tunnel $\partial$-reducing knots is also given in section 3. It is easy to see that the dual knot of a $\partial$-reducing 1-bridge knot may not be 1-bridge. However, we have

Theorem 4 If $K$ is a $\partial$-reducing 1-tunnel knot, then its dual with respect to a $\partial$-reducing surgery is also a 1-tunnel knot.

As a corollary, we have

Theorem 5 Suppose $K$ is a 1-bridge knot in a handlebody $M$ such that some nontrivial surgery on $K$ produces a handlebody $M^{\prime}$. Then the dual knot $K^{\prime}$ in $M^{\prime}$ is also a 1-bridge knot.

Part of this work was done while I was visiting the University of Texas at Austin. I would like to thank John Berge, Cameron Gordon and John Luecke for some interesting conversations on this topic.

Notations and definitions. We work on smooth category. All 3-manifolds $M$ are assumed orientable. For a curve $c$ in $\partial M$, let $\tau(M, c)$ be the manifold obtained from $M$ by attaching a 2-handle along $c$. If $X$ is a subset of $M, N(X)$ will stand for a regular neighborhood of $N$. For a knot $K$ in $M$, and $\gamma$ a slope on $\partial N(K)$, denote by $(M, K ; \gamma)$ the manifold obtained by Dehn surgery on $K$ with slope $\gamma$. That is, 
$(M, K ; \gamma)=(M-\operatorname{Int} N(K)) \cup\left(S^{1} \times D^{2}\right)$, where $\partial\left(S^{1} \times D^{2}\right)$ is identified with $\partial N(K)$ so that some $\{$ point $\} \times \partial D^{2}$ is identified with $\gamma$. Let $K^{\prime}$ be the center curve of the attached solid torus. We call $K^{\prime}$ the dual of $K$ with respect to this surgery.

A properly embedded surface $S$ in a 3 -manifold $M$ is called essential if it incompressible and $\partial$-incompressible. Suppose $D$ is an embedded disk in $M$ such that either $D \cap F=\partial D$, or $D \cap F$ is an arc $\alpha$ in $\partial D$ and the rest part of $\partial D$ lies on $\partial M$. Choose a product $D \times I$ in $M$ so that $F \cap(D \times I)=(F \cap \partial D) \times I$. The surface $F^{\prime}=(F-\partial D \times I) \cup(D \times \partial I)$ is called obtained from $F$ by surgery along $D$.

\section{$1 \quad \partial$-Reducing surgeries on satellite knot}

A knot in $M$ is called a satellite knot (or more precisely, a satellite of $L$ ) if there is a nontrivial knot $L$ in $M$ and a regular neighborhood $N(L)$ of $L$ containing $K$, such that (i) $L$ is not the central curve of $M$ if $M$ is a solid torus; (ii) $K$ is not isotopic to $L$; and (iii) $\partial N(L)$ is incompressible in $N(L)-K$. In this section we will give some positive results on the 1-bridge conjecture. Especially, it is true for knots in solid tori, as well as for satellite knots in 3-manifolds. The following are examples of $\partial$-reducing satellite knots.

Example 1. Let $K_{1}$ be a 0 -bridge knot in $M$. We assume that $\partial M$ is compressible in $M$, and incompressible in $M-K$. Let $m$ be a meridian on $\partial N(K)$, and let $l \subset \partial N(K)$ be a boundary component of an annulus $A$ embedded in $M-\operatorname{Int} N\left(K_{1}\right)$ whose other boundary lies on $\partial M$. The pair $(m, l)$ is called a preferred meridian-longitude pair on $\partial N\left(K_{1}\right)$. Let $K$ be a $(p, 1)$ cable $(p>1)$ of $K_{1}$, i.e. $K$ is a simple closed curve on $\partial N\left(K_{1}\right)$ representing $p l+m$ in homology. Note that $K$ is a 1-bridge knot in $M$. Let $\gamma$ be the cabling slope on $\partial N\left(K_{1}\right)$. Then surgery on $K$ with slope $\gamma$ will produce $M^{\prime}=M \# L(p)$, where $L(p)$ is a lens space with $\pi_{1}=\mathbf{Z}_{p}$. Therefore $K$ is a $\partial$-reducing knot. Note that its dual $K^{\prime}$ is also a $\partial$-reducing knot, but is not a 1-bridge knot because it has homotopically nontrivial intersection with $L(p)$, and hence is not homotopic to a curve on $\partial M$.

We will show that these are essentially all the possible satellite $\partial$-reducing knots.

Let $N$ be a solid torus, and let $(m, l)$ be a meridian-longitude pair on $\partial N$. Suppose $K$ is a knot in $N$ with winding number $p$. Choose a meridian-longitude pair $(\bar{m}, \bar{l})$ on $\partial N(K)$ so that homologically $m=p \bar{m}$, and $\bar{l}=p l$. If $L$ is a 0 -bridge $\operatorname{knot}$ in $M$, we choose the meridian-longitude $(m, l)$ on $\partial N(L)$ so that $l$ is a boundary component of an 
embedded annulus $A$ in $M-\operatorname{Int} N(L)$ whose other end lies on $\partial M$, and call $(m, l)$ a preferred meridian-longitude pair of $L$.

Lemma 1.1 Suppose $K$ is a 1-bridge (probably 0-bridge) knot in a solid torus $N$ with winding number $p>1$, and suppose some nontrivial surgery on $K$ produces a solid torus $N^{\prime}=(N, K ; \gamma)$. Let $m^{\prime}$ be a meridian of $N^{\prime}$. Then $\Delta\left(m, m^{\prime}\right)>1$, and either (i) $\Delta\left(l, m^{\prime}\right)>$ 1 , or (ii) $K$ is a $(2, \pm 1)$ cable of the central curve of $N$, and $\gamma=\bar{l} \pm \bar{m}$.

Proof. Suppose $\gamma=a \bar{l}+b \bar{m}$. Since $\gamma \neq \bar{m}$, we may assume $a>0$. By [6, Lemma 3.3], $m^{\prime}=a p^{2} l+b m$. If $K$ is a $(p, q)$ cable of the central curve of $N$, then by [6, Lemma 7.2], $(a, b)=(n, n p q \pm 1)$ for some $n>0$. Therefore, $\Delta\left(m, m^{\prime}\right)=\left|a p^{2}\right|=n p^{2}>1$, and $\Delta\left(l, m^{\prime}\right)=|n p q \pm 1|>1$ unless $n=1, p=2$ and $q= \pm 1$, in which case $\gamma=l \pm m$, as required.

If $K$ is 1-bridge but not 0-bridge, then by [5, Lemma 3.2], $\gamma=\bar{l}+b \bar{m}$ for some $b \neq 0$. Since $K$ is not 0 -bridge, $p \geq 3$. So by [11, Lemma 3], $b \neq \pm 1$. Since $m^{\prime}=p^{2} l+b m$, we have $\Delta\left(m, m^{\prime}\right)=p^{2}>1$ and $\Delta\left(l, m^{\prime}\right)=|b|>1$.

Theorem 1 Suppose $K$ is a $\partial$-reducing knot in a 3-manifold $M$ with $M-K$ irreducible, and suppose there is a torus $T$ which separates $K$ from $\partial M$ but does not bound a regular neighborhood of $K$. Then either $M$ is a solid torus and $K$ is a 1-bridge braid, or one of $K$ and $K^{\prime}$ is a $(p, \pm 1)$ cable $(p>1)$ of some 0-bridge knot $L$ with respect to some preferred meridian-longitude pair. Especially, either $K$ or $K^{\prime}$ is a 1-bridge knot.

Proof. Since $T$ separates $K$ from $\partial M, T$ bounds a 3-manifold $N$ containing $K$. By Haken's finiteness theorem [8, Lemma 13.2], we may choose $T$ so that there is no essential tori in $N$ separating $K$ from $T$. Since $\partial M$ is incompressible in $M-K$, it is incompressible in $X=M-\operatorname{Int} N$. The surgered manifold $M^{\prime}=(M, K ; \gamma)$ can be written as $X \cup_{T}(N, K ; \gamma)$. If $T$ is incompressible in $M$ or $M^{\prime}$, then a compressing disk of $\partial M$ can be modified into one which is disjoint from $T$, which is impossible because $\partial M$ is incompressible in $M-K$. Thus $\partial N$ is compressible in both $N$ and $N^{\prime}=(N, K ; \gamma)$. It follows that $N$ is either a solid torus or is reducible. The same is true for $N^{\prime}$. There are two cases.

CASE 1: Both $N$ and $N^{\prime}$ are solid tori. 
In this case, $K$ is a 1-bridge braid in $N$ with winding number $p>1$. (See [4]). Denote by $L$ the central curve of $N$. Let $m$ (resp. $m^{\prime}$ ) be the meridian of $N$ (resp. $N^{\prime}$ ). Then $M=X \cup_{T} N=(M, L ; m)$ and $M^{\prime}=X \cup_{T} N^{\prime}=\left(M, L ; m^{\prime}\right)$. According to Lemma 1.1, we always have $\Delta\left(m, m^{\prime}\right)>1$. Because $\partial M$ is compressible in both $M$ and $M^{\prime}, L$ is a 0-bridge knot in $M$ [12, Thm 2]. It follows from [3, Thm 2.4.3] that either $M$ is a solid torus and $L$ is the central curve, in which case $K$ is a 1-bridge braid in $M$, or $\Delta\left(l, m^{\prime}\right) \leq 1$, where $l$ is a preferred longitude of $L$. By the lemma above the latter is possible only if $K$ is a $(2, \pm 1)$ cable of $L$.

CASE 2: Either $N$ or $N^{\prime}$ is reducible.

Scharlemann [10] showed that if $N$ is $\partial$-reducible and $N^{\prime}$ is reducible, then $K$ is a cable knot, and the surgery slope is that of the cabling slope. Therefore if $N^{\prime}$ is reducible, then $K$ is a cable knot, and $\gamma$ is the slope of the cabling annulus. Now the assumption that there is no essential tori in $N$ separating $K$ from $T$ implies that $N$ is a solid torus and $K$ is a $(p, q)$ cable $(p>1)$ of the central curve $L$ of $N$. We have $M^{\prime}=X \cup N^{\prime}$, where $N^{\prime}=(N, K ; \gamma)=N^{\prime \prime} \# L(p, q)$, and $N^{\prime \prime}$ is a solid torus whose meridian $m^{\prime}$ is a $(p, q)$ curve (i.e., homologous to $p l+q m)$ on $T$. Especially, $M^{\prime}=\left(M, L ; m^{\prime}\right) \# L(p, q)$, and $\Delta\left(m, m^{\prime}\right)=p>1$. Again by [12, Thm 2] and [3, Thm 2.4.3], $L$ is a 0-bridge knot, and $\Delta\left(l, m^{\prime}\right) \leq 1$. Since $\Delta\left(l, m^{\prime}\right)=|q|$, we have that $K$ is a $(p, \pm 1)$ cable of the 0 -bridge knot $L$.

Similarly, if $N$ is reducible, then $K^{\prime}$ is a $(p, \pm 1)$ cable of some 0 -bridge knot in $M^{\prime}$.

Corollary 1.2 If $K$ is a knot in a solid torus $M$ with nonzero wrapping number, and if some nontrivial surgery on $K$ results in a $\partial$-reducible manifold, then $K$ is a 1-bridge braid in $M$.

Proof. A torus parallel to $\partial M$ separates $K$ from $\partial M$, so we can apply Theorem 1 . If $K$ is a $(p, \pm 1)$ cable of some 0 -bridge knot $L$ in $M$, then since $K$ has nonzero wrapping number, $L$ is not a meridian or trivial knot. So $K$ is a 1-bridge braid. Similarly, if $K^{\prime}$ is a $(p, \pm 1)$ cable of some 0 -bridge knot, then $K^{\prime}$ is a 1-bridge braid. Since $M=\left(M^{\prime}, K^{\prime} ; m\right)$ is a solid torus, by [4, Thm 1.1] $K$ is also a 1-bridge braid.

Corollary 1.3 Suppose $K$ is a satellite knot in a 3-manifold $M$ with $M-K$ irreducible. If $K$ is $\partial$-reducing, then it is a $(p, \pm 1)$ cable of some 0-bridge knot. Especially, it is a 1-bridge knot. 
Proof. Suppose $K$ is a satellite of $L$ and let $N=N(L)$ be a regular neighborhood of $L$ containing $K$. As in the proof of Theorem 1, we may assume there is no essential tori in $N$ separating $K$ from $\partial N$. Examine the proof of Theorem 1 . If both $N$ and $N^{\prime}=(N, K ; \gamma)$ are solid tori, then, since $L$ is not a central curve when $M$ is a solid torus (by definition of satellite knot), the conclusion of Case 1 asserts that $K$ is a $(2, \pm 1)$ cable of $L$ and $L$ is a 0 -bridge knot. In Case 2 , since $N$ is irreducible, $N^{\prime}$ is reducible. So the proof there shows that $K$ is a $(p, \pm 1)$ cable of $L, p>1$, and $L$ is a 0 -bridge knot.

Remark. By the proof, $\partial$-reducing surgeries are also clear: Suppose $K$ is a $(p, \pm 1)$ cable of a 0 -bridge knot $L$. If $p \geq 3$, there is exactly one nontrivial $\partial$-reducing surgery, i.e. the surgery along the cabling slope. If $p=2$, there are exactly two nontrivial $\partial$-reducing surgeries: One is along the cabling slope, another has slope $\gamma=\bar{l} \pm \bar{m}$ given by Lemma 1.1 .

\section{A knot that is $\partial$-reducing but not 1-tunnel}

Let $H$ be a handlebody of genus 2. Choose simple closed curves $c_{1}, c_{2}$ on $\partial H$ so that $c_{i}$ runs around the $i$-th handle twice, and is disjoint from the other handle. Let $Y_{i}(i=1,2)$ be a compact 3-manifold with boundary an incompressible torus, and let $c_{i}^{\prime}$ be an essential simple closed curve on $\partial Y_{i}$. Denote by $A_{i}$ (resp. $A_{i}^{\prime}$ ) a regular neighborhood of $c_{i}$ (resp. $c_{i}^{\prime}$ ). Identifying $A_{i}$ with $A_{i}^{\prime}$, we get a 3 -manifold $M$ with $A_{i}$ a properly embedded annulus. By the construction it is easy to see that $A_{i}$ are essential (i.e. incompressible and $\partial$ incompressible) in $M$. Let $K$ be a simple loop in $\operatorname{Int} H$ which runs once around each handle. The purpose of this section is to show that $K$ is a $\partial$-reducing knot but not a 1-tunnel knot in $M$. (See Figure 1).

(Figure 1)

Lemma 2.1 K admits a $\partial$-reducing surgery.

Proof. There is an annulus $Q$ as shown in Figure 1, which has one end on $\partial H$ and the other on $\partial N(K)$. There is a self homeomorphism $\varphi$ of $H-\operatorname{Int} N(K)$ obtained by cutting 
$H$ along $Q$, making a full twist, then gluing back. This does not change $H-\operatorname{Int} N(K)$, but it changes the slopes on $\partial N(K)$. Let $m$ be a meridian on $\partial N(K)$. Then $\gamma=\varphi^{-1}(m)$ is a nontrivial slope on $\partial N(K)$, and $\varphi$ extends to a homeomorphism $\varphi:(H, K ; \gamma) \rightarrow H$. It follows that the surgery does not change $H$, but it changes the curves $c_{i}$ into $\bar{c}_{i}=\varphi\left(c_{i}\right)$. Note that $M^{\prime}=(M, K ; \gamma)$ can be obtained by identifying $A_{i}^{\prime}$ with $\varphi\left(A_{i}\right)$ on $\partial H$, and $\varphi\left(A_{i}\right)$ is a neighborhood of $\bar{c}_{i}$. Figure 2(a) indicates the curves $\bar{c}_{i}$; Figure 2(b) is a simplification of 2(a). Turning one of the handle around by a half twist, we get Figure 2(c). It is now clear that the curve $J^{\prime}$ in Figure 2(c) bounds a compressing disc of $H$ disjoint from $\bar{c}_{i}$. Therefore $\partial M^{\prime}$ is compressible.

(Figure 2)

Denote by $K^{\prime}$ the dual knot with respect to the above $\partial$-reducing surgery, and by $D^{\prime}$ the disk bounded by $J^{\prime}$ in Figure 2(c). Corollary 2.3 and Lemma 2.4 are proved for $M$, but they are are still true when replace $K, D, H, M$ by $K^{\prime}, D^{\prime}, H^{\prime}, M^{\prime}$, respectively.

Lemma 2.2 Suppose $F_{1}, F_{2}$ are disjoint incompressible surfaces on the boundary of an irreducible 3-manifold $N$. Let $D_{i}$ be a disk in $\operatorname{Int} F_{i}$, and let $H$ be obtained from $N$ by identifying $D_{1}$ and $D_{2}$ into a single disk $D$. Then up to proper isotopy $D$ is the unique compressing disk of $F=\left(F_{1} \cup F_{2}\right)-\operatorname{Int} D$ in $H$.

Proof. This is a standard innermost circle outermost arc argument. We omit the details.

Corollary 2.3 Up to proper isotopy $D$ is the only compressing disk of $F$ in $H$, and hence the only compressing disk of $\partial M$ in $M$.

Proof. Cut $H$ along $D$, we get a manifold $N$. The surface $\partial N-\operatorname{Int}\left(A_{1} \cup A_{2}\right)$ consists of two annuli $F_{1}$ and $F_{2}$ with $\partial F_{i}=\partial A_{i}$. Since $A_{i}$ is incompressible, $F_{i}$ is incompressible. So by Lemma 2.3, $D$ is the unique compressing disk of $F$ in $H$. Since $H$ and $Y_{i}$ are irreducible, and $A_{i}$ is essential, any compressing disk $D^{\prime}$ of $\partial M$ can be isotoped off $A_{i}$, and therefore lies in $H$ because $\partial Y_{i}$ is incompressible. It follows that $D^{\prime}$ is properly isotopic to $D$ in $M$. 
Lemma 2.4 Suppose $K$ is homotopic to some curve $L=\alpha \cup \beta$ in $M$ such that $\alpha \subset \partial M$, and $\beta \cap D=\emptyset$. Then $L$ can be chosen to be disjoint from $A=A_{1} \cup A_{2}$, and $K$ is homotopic to $L$ in $H$.

Proof. Among all curves $L$ satisfying the hypothesis, choose one such that $|L \cap A|$ is minimal, where $|X|$ denotes the number of components in $X$. Let $\varphi: S^{1} \times I \rightarrow M$ be a map such that $\varphi\left(S^{1} \times 0\right)=K$, and $\varphi\left(S^{1} \times 1\right)=L$. We may assume that $\varphi$ is transverse to $A$. Since $K \cap A=\emptyset$, an arc component of $\varphi^{-1}(A)$ has both ends at $S^{1} \times 1$. Let $s$ be an outermost arc. Then there is an arc $s^{\prime}$ in $S^{1} \times 1$, such that $s \cup s^{\prime}$ bounds a disk $P$ in $S^{1} \times I$. Especially, $\gamma^{\prime}=\varphi\left(s^{\prime}\right) \subset L$ is rel $\partial \gamma^{\prime}$ homotopic to $\gamma=\varphi(s)$ in $A$, so $K$ is homotopic to $L^{\prime}=\left(L-\gamma^{\prime}\right) \cup \gamma$.

The disk $P$ gives rise to a homotopy $\gamma \sim \gamma^{\prime}(\operatorname{rel} \partial \gamma)$. Since $A$ is essential, $\gamma$ is an inessential proper curve on $A$. If $\gamma^{\prime} \cap \beta=\emptyset$, then $\gamma$ is contained in $\alpha$. It is rel $\partial \gamma$ homotopic to some $\operatorname{arc} \gamma^{\prime \prime}$ on $\partial A$. Let $\alpha^{\prime}=\left(\alpha-\gamma^{\prime}\right) \cup \gamma^{\prime \prime}$. Then $K$ is homotopic to $\alpha^{\prime} \cup \beta$, and a further homotopy will reduce $|L \cap A|$. If $\beta \cap \gamma^{\prime} \neq \emptyset$, then $\alpha^{\prime}=\alpha-\operatorname{Int} \gamma^{\prime}$ and $\beta^{\prime}=\beta \cup \gamma$ are connected, $\alpha^{\prime} \subset \partial M$, and $\beta^{\prime} \cap D=\gamma \cap D \subset A \cap D=\emptyset$. A perturbation of $L^{\prime}=\alpha^{\prime} \cup \beta^{\prime}$ will satisfy the hypothesis of the lemma, but lower $|L \cap A|$. Both cases contradict the minimality of $|L \cap A|$. therefore no such arc $\gamma$ exists, which implies $L \cap A=\emptyset$.

Now let $s$ be a circle component of $\varphi^{-1}(A)$. If $s$ is essential in $S^{1} \times I$, then $K$ is homotopic to $\varphi(s)$ in some $A_{i}$, which is impossible due to homological reasons. If $s$ is inessential, then it bounds a disk $P$ in $S^{1} \times I$. Since $A$ is incompressible, $\varphi(s)$ is null homotopic, so we can change $\varphi$ by mapping $P$ into $A$, then pushing off $A$. This will reduce $\left|\varphi^{-1}(A)\right|$. Eventually $\varphi$ will be disjoint from $A$, and hence is a homotopy in $H$.

Lemma $2.5 K$ and $K^{\prime}$ are not 1-tunnel knots.

Proof. By Corollary 2.3, $D$ is the unique compressing disk of $\partial M$. So if $K$ is 1-tunnel, then $K$ is homotopic to some $L=\alpha \cup \beta$ with $\alpha \subset \partial M$ and $\beta \cap D=\emptyset$. By Lemma 2.4, we can further assume that $\alpha \subset F, \beta \subset H$, and $K$ is homotopic to $L$ in $H$.

The disk $D$ separates $H$ into two solid tori $H_{1}$ and $H_{2}$. Since $\beta$ is disjoint from $D$, it lies in one of the solid tori, say $H_{1}$. Let $f: H \rightarrow H_{2}$ be a retraction which maps $H_{1}$ into $D$. Then $f(K)$ is homotopic to $f(L)$ in $H_{2}$. This is obviously impossible because $f(K)$ is homotopic to the central curve of $H_{2}$, while $f(L)$ lies on $\partial H_{2}-c_{2}$. 
Now consider $K^{\prime}$. As above, we assume $K^{\prime}$ is homotopic to $\alpha \cup \beta$, where $\alpha \subset F$, and $\beta$ is disjoint from $D^{\prime} \subset M^{\prime}$. (See Figure 2(c)). The algebraic intersection number of $K$ and $D$ is 2 . Since $\beta$ is disjoint from $D^{\prime}, \alpha$ must have intersection number 2 with $D^{\prime}$. It follows that $\partial D^{\prime}$ is nonseparating on $F$. But this is impossible because $F$ is a 2 -sphere with four holes.

Theorem 2 There is a compact 3-manifold $M$ and a $\partial$-reducing knot $K$ in $M$, such that neither $K$ nor its dual $K^{\prime}$ with respect to the $\partial$-reducing surgery are 1-tunnel knots.

Proof. By Lemma 2.1, $K$ admits a $\partial$-reducing surgery. By Lemma 2.5, $K$ and $K^{\prime}$ are not 1-tunnel knots. It is quite clear that $K$ can not be isotoped off $D$. Since $D$ is the unique compressing disk, $\partial M$ is incompressible in $M-K$.

\section{Surgery on 1-tunnel knots}

We have seen that a $\partial$-reducing knot in a general 3-manifold may not be a 1-tunnel knot, but such a knot in a solid torus must be a 1-bridge knot. An interesting problem is if this is true for handlebodies of genus greater than 1 .

Conjecture A. A $\partial$-reducing knot in a handlebody is a 1-bridge knot.

Conjecture B. If a knot in a handlebody admits a nontrivial surgery so that the resulting manifold is still a handlebody, then it is a 1-bridge knot.

Clearly, Conjecture A is stronger than Conjecture B. These are probably Berge's original conjectures. In this section, we will show that Conjecture A is true if $K$ is a 1-tunnel knot. This gives new evidence to the conjectures. It follows that Conjecture A is equivalent to the weaker statement that $\partial$-reducing knot in a handlebody is a 1-tunnel knot.

Lemma 3.1 Let $K$ be a $\partial$-reducing knot in a handlebody $M$. If $\partial M$ has a compressing disc $D$ intersecting $K$ at one point, then $M$ is a solid torus, and $K$ is isotopic to the central curve of $M$.

Proof. If $M$ is a solid torus, then by Corollary $1.2, K$ being $\partial$-reducing implies $K$ is a 1-bridge braid. The hypothesis now implies that $K$ is isotopic to the central curve of $M$. So we assume $M$ has genus at least 2 . 
Consider the exterior $X=M-\operatorname{Int} N(K)$ of $K$. The annulus $A=D \cap X$ has one boundary component on $\partial M$, and the other on $\partial N(K)$ with slope the meridian $m$ of $K$. By definition of $\partial$-reducing knot, $\partial M$ is incompressible in $X$. So Theorem 2.4.3 of [3] implies that if $\partial M$ is compressible in $M^{\prime}=(M, K ; \gamma)(=X(\gamma)$ by notation of [3]), then either $\gamma=m$, which is the trivial surgery, or $\Delta(m, \gamma)=1$, in which case the dual knot $K^{\prime}$ is a boundary parallel knot, and $A$ extends to an annulus with a boundary component on $K^{\prime}$. Consider $X$ as a subset of $M^{\prime}$. It is now easy to see that cutting $X$ along $A$ produces a manifold $W=X-\operatorname{Int} N(A)$ homeomorphic to $M^{\prime}$. Especially, $\partial W$ is compressible.

Now consider $W$ as a subset of $M$. We have $W=X-\operatorname{Int} N(A)=M-\operatorname{Int} N(K \cup D)$. Notice that $\partial W-\partial M=\partial N(K \cup D)-\partial M$ is an annulus. Let $s$ be a central curve of this annulus. Then $M$ can be reconstructed from $W$ by attaching a 2-handle along $c$ to obtain $\tau(W, c)=M-\operatorname{Int} N(D)$, then gluing in $N(D)$.

The subsurface $S=W \cap \partial M$ of $\partial M$ has no boundary component bounding disks on $\partial M$. Since $\partial M$ is incompressible in $M-K, S$ is also incompressible in $M-K$; so $S$ is incompressible in $W$. Since $S$ is isotopic to $\partial W-c$ on $\partial W, \partial W-c$ is incompressible in $W$. It has been shown above that $\partial W$ is compressible. Therefore, by Jaco's Handle Addition Theorem [9, Lemma 1], the manifold $\tau(W, c)=M-\operatorname{Int} N(D)$ has incompressible boundary. This is impossible because $M-\operatorname{Int} N(D)$ is a handlebody of genus $g-1 \geq 1$.

Lemma 3.2 Suppose $\alpha \cup \beta$ is a 1-tunnel presentation of $K$ in a 3-manifold $M$. Let $W=$ $M-\operatorname{Int} N(\beta)$, and let $m$ be the meridian slope of $K$. If $\Delta(\gamma, m)=1$, then $(M, K ; \gamma)$ is homeomorphic to $\tau(W, c)$ for some $c=\alpha^{\prime} \cup \delta$, where $\alpha^{\prime}=\alpha \cap \partial W$, and $\delta$ is an essential arc on the annulus $A=W \cap N(\beta)$.

Proof. Construct a pair $\left(M^{\prime}, K^{\prime}\right)$ as follows. Let $H=N(\beta)$ be a regular neighborhood of $\beta$. Attach a 1-handle $H^{\prime}=D^{2} \times I$ to $M$ so that $D^{2} \times \partial I$ is identified with the two disks in $H \cap \partial M$. Call this manifold $Y$. The neighborhood $H$ of $\beta$ can be chosen so that $\alpha^{\prime}=\alpha \cap \partial Y$ is an arc. Let $\delta^{\prime}$ be an arc on $\partial H^{\prime}$ connecting the two ends of $\alpha^{\prime}$. Then $c^{\prime}=\alpha^{\prime} \cap \gamma^{\prime}$ is a simple closed curve on $\partial Y$ intersecting the meridian $D^{2} \times\{$ point $\}$ of $H^{\prime}$ at one point. Therefore, when attaching a disk to $Y$ along $c$, it cancels the 1-handle $H^{\prime}$; so the resulting manifold $M^{\prime}=\tau(Y, c)$ is homeomorphic to $M$. Let $\beta^{\prime}$ be the central arc $0 \times I$ in $H^{\prime}$, and let $K^{\prime}=\beta \cup \beta^{\prime}$. Since $K^{\prime}$ is isotopic to $\alpha \cup \beta$ in $M^{\prime}$, the homeomorphism 
$M \cong M^{\prime}$ can be chosen so that $K$ is mapped to $K^{\prime}$, i.e. we have a homeomorphism of pairs $(M, K) \cong\left(M^{\prime}, K^{\prime}\right)$. Denote by $\gamma^{\prime}$ the slope on $\partial N\left(K^{\prime}\right)$ corresponding to $\gamma$.

Let $N$ be the solid torus $H \cup H^{\prime}$. Denote by $A$ the annulus $H \cap W$, and by $A^{\prime}$ the annulus $\partial D^{2} \times I$ on $\partial H^{\prime}$. Then $Y=W \cup_{A} N$. Writing $N_{1}=\left(N, K^{\prime} ; \gamma^{\prime}\right)$, we get a homeomorphism

$$
\left(Y, K^{\prime} ; \gamma^{\prime}\right)=W \cup_{A}\left(N, K^{\prime} ; \gamma^{\prime}\right)=W \cup_{A} N_{1}
$$

It means that we can perform the surgery on $K^{\prime}$ in $N$, then glue the resulting manifold to $W$ along the annulus $A$ to obtain $\left(Y, K^{\prime} ; \gamma^{\prime}\right)$.

The knot $K^{\prime}$ is the central curve in the solid torus $N$; so after surgery $N_{1}=\left(N, K^{\prime} ; \gamma^{\prime}\right)$ is still a solid torus. The central curve $s$ of the annulus $A$ is isotopic to a meridian $m^{\prime}$ of $K^{\prime}$. Since $\Delta\left(m^{\prime}, \gamma^{\prime}\right)=\Delta(m, \gamma)=1$, after surgery $s$ is a longitude on $\partial N_{1}$. Therefore, when gluing $N_{1}$ to $\mathrm{W}$ along $A$, the resulting manifold $W \cup_{A} N_{1}$ is homeomorphic to $W$ itself. Under this homeomorphism, the annulus $A^{\prime}=\partial D^{2} \times I$ in $H^{\prime}$ is mapped to $A$, and the curve $c^{\prime}$ is mapped to $c=\alpha^{\prime} \cup \delta$, for some $\operatorname{arc} \delta$ in $A$. We have $M \cong M^{\prime} \cong \tau\left(Y, c^{\prime}\right)$; so

$$
(M, K ; \gamma)=\left(M^{\prime}, K^{\prime} ; \gamma^{\prime}\right)=\left(\tau\left(Y, c^{\prime}\right), K^{\prime} ; \gamma^{\prime}\right)=\tau\left(\left(Y, K^{\prime} ; \gamma^{\prime}\right), c^{\prime}\right) \cong \tau(W, c)
$$

The third equality follows by exchanging the order of 2-handle addition and Dehn surgery. This completes the proof.

Lemma 3.3 Let $\alpha, \beta, W$ be as in Lemma 3.2 and assume $M$ is a handlebody. If $K$ is not a 1-bridge knot, then there is a closed incompressible surface $F \subset \operatorname{Int} W$, and an incompressible annulus $P$ in $W$ with one boundary component $\partial_{0}$ on $F$, and the other one $\partial_{1}$ on the annulus $A=W \cap N(\beta)$.

Proof. Cut $W$ along compressing disks of $\partial W-A$ until we get a manifold $W^{\prime}$ such that $\partial W^{\prime}-A$ is incompressible, where $A$ is the annulus $W \cap N(\beta)$. Let $W^{\prime \prime}$ be the component of $W^{\prime}$ containing $A$. It is quite clear that $A$ is incompressible in $W$, otherwise $M$ would be reducible. Therefore $\partial W^{\prime \prime}$ is not a 2 -sphere. We want to show that $\partial W^{\prime \prime}$ is incompressible.

If $\partial W^{\prime \prime}$ is compressible, then, since $\partial W^{\prime \prime}-J$ is incompressible, by the Handle Addition Theorem [9], $M^{\prime \prime}=W^{\prime \prime} \cup N(\beta)=\tau\left(W^{\prime \prime}, J\right)$ has incompressible boundary. Since $M^{\prime \prime}$ is a component of the manifold obtained from $M$ by cutting along some disks, it is a handlebody. So $\partial M^{\prime \prime}$ being incompressible implies that $M^{\prime \prime}$ is a 3 -ball. If $\beta$ is knotted, 
then $\partial W^{\prime \prime}$ is incompressible. If $\beta$ is unknotted then one can find an $\operatorname{arc} \beta^{\prime}$ on $\partial W^{\prime \prime}$ such that $\beta^{\prime} \cup \beta$ bounds a disk. Since $\partial M^{\prime \prime}-\partial M$ are disks, by an isotopy rel $\partial \beta^{\prime}$ we may assume that $\beta^{\prime} \subset \partial M$. This contradicts the hypothesis that $K$ is not a 1-bridge knot. Therefore, $\partial W^{\prime \prime}$ is always incompressible.

Let $F$ be $\partial W^{\prime \prime}$ pushing into $W^{\prime \prime}$, and let $P$ be an annulus in $W^{\prime \prime}$ with one boundary component on $F$ and the other equal to $J$ on $A$. Then $P$ and $F$ are obviously incompressible in $W^{\prime \prime}$, and hence in $W^{\prime}$. Since $W$ is obtained from $W^{\prime}$ by attaching some 1-handles, $F$ and $P$ remain incompressible in $M$.

Theorem 3 If $K$ is a $\partial$-reducing 1-tunnel knot in a handlebody $M$, then it is a 1-bridge knot.

Proof. We may assume that $K$ is not boundary parallel, otherwise it is a 1-bridge knot. By [12, Thm 2], if $(M, K ; \gamma)$ is a nontrivial $\partial$-reducing surgery, then $\Delta(m, \gamma)=1$; so by Lemma $3.2,(M, K ; \gamma)$ is homeomorphic to some $\tau(M, c)$, where $W=M-\operatorname{Int} N(\beta)$, and $c$ is a certain curve on $\partial W$. There is, by definition of 1-tunnel knot, a compressing disk $D$ of $\partial M$ disjoint from $\beta$. $D$ remains a compressing disk of $\partial W$ in $W$, so $\partial W$ is compressible. In the following, we will show that if $K$ is a 1-bridge knot, then $\partial W-c$ is incompressible. It would then follow from the Handle Addition Theorem [9] that $\tau(W, c)$ has incompressible boundary, contradicting the assumption that $(M, K ; \gamma) \cong \tau(W, c)$ is the result of a $\partial$-reducing surgery.

Suppose $K$ is not a 1-bridge knot. We need to show that $\partial W-c$ is incompressible. Apply Lemma 3.3, and let $F$ and $P$ be the incompressible surfaces given there. Since $F$ is a closed incompressible surface, we can isotop $D$ off $F$. So we assume $D$ is disjoint from $F$. Consider $D \cap P$ in the annulus $P$. No component of $D \cap P$ is an essential circle in $P$ because $P$ is incompressible. Surgering $D$ along a disk in $P$ bounded by an innermost circle of $D \cap P$ will reduce $|D \cap P|$, so we assume that $D \cap P$ are $\operatorname{arcs}$. Since $D$ is disjoint from $F$, any arc component of $D \cap P$ has both ends on $\partial_{1}=P \cap A$, where $A$ is the annulus $N(\beta) \cap W$. There are three cases:

Case 1. Some arc $s$ cuts off a disk $B$ in $P$ disjoint from $c$.

Choose an outermost such arc $s$. Surgery of $D$ along $B$ produces two new disks $D_{1}, D_{2}$, one of which is a compressing disk of $\partial W-c$, but has fewer components of intersection with $A$. Repeating this process will eliminate all such intersections. 
CASE 2. $D \cap P \neq \emptyset$, and any arc $s$ in $D \cap P$ cuts off a disk $B$ in $P$ which intersects $c$.

Recall that $c=\alpha^{\prime} \cup \delta$, where $\alpha^{\prime}=\alpha \cap \partial W$, and $\delta \subset A$. So we can assume that $\delta$ intersects $P$ at a single point, and hence $B$ intersects $\delta$ at a single point.

Let $s$ be an arc of $D \cap P$ which is outermost in $D$ (rather than in $P$ ), and let $D_{1}$ be the outermost disk in $D$ cut off by $s$. We have $P \cap \operatorname{Int} D_{1}=\emptyset$. Consider the disk $D^{\prime}=D_{1} \cup B$ enbedded in $W$, where $B$ is, as above, the disk in $P$ cut off by $s$. Since $P \cap \operatorname{Int} D_{1}=\emptyset$, we can isotop $D^{\prime}$ off $P$. Especially, it is disjoint from $\partial_{1}=A \cap P$. Since $\partial_{1}$ is the central curve of $A$, another isotopy will move $D^{\prime}$ off $A$ to a disk $D^{\prime \prime}$. Furthermore, because $C \cap A$ is a proper arc, and $\left|D^{\prime} \cap c\right|=1$, we may assume that $D^{\prime \prime}$ intersects $\alpha$ at a single point. Especially, it is nonseparating, and so is a compressing disk of $\partial M$. Since $D^{\prime \prime}$ intersects $\alpha$ once, and is disjoint from $\beta$, it intersects $K$ at a single point. By Lemma 3.1, $M$ is a solid torus, and $K$ is the central curve. This contradicts the assumption that $K$ is not a 1-bridge knot.

CAse 3. $D$ is disjoint from P. Especially, $\partial D \subset W-\left(c \cup \partial_{1}\right)$.

By an isotopy, we may assume that $\partial D$ is disjoint from $c \cup A$. Such a disk is a compressing disk of $\partial M-\alpha$ in $M-(\alpha \cup \beta)$. Consider $K$ as $\alpha \cup \beta$ pushing slightly into Int $M$. Then $D$ is a compressing disk of $\partial M-\alpha$ in $M-K$. If $\partial D$ is essential in $\partial M$, then it is a compressing disk of $\partial M$ in $M-K$. If $\partial D$ is inessential in $\partial M, \partial D$ bounds a disk $D^{\prime}$ containing $\alpha$, so $D \cup D^{\prime}$ bounds a 3-cell containing $K$. Hence any compressing disk of $\partial M$ in $M$ can be pushed off $K$ and becomes a compressing disk of $\partial M$ in $M-K$. Both cases contradict the fact that $\partial M$ is incompressible in $M-K$.

As we noticed in the introduction part, for a 1-bridge knot $K$, its dual with respect to a $\partial$-reducing surgery may not be 1-bridge. But the following result shows that the "1-tunnel" property is invariant under $\partial$-reducing surgeries.

Theorem 4 If $K$ is a $\partial$-reducing 1-tunnel knot, then its dual with respect to a $\partial$-reducing surgery is also a 1-tunnel knot.

Proof. Suppose $M^{\prime}=(M, K ; \gamma)$ is a $\partial$-reducing surgered manifold, and let $m$ be the meridian slope of $K$. If $\Delta(m, \gamma)>1$, then by [12, Thm 2] and [3, Thm 2.4.3], $K^{\prime}$ is either boundary parallel, and hence 1-tunnel, or intersects a compressing disk $D$ of $\partial M^{\prime}$ at one point. In the later case, we can push a small arc in a neighborhood of $D$ into $\partial M^{\prime}$, getting a 1-tunnel presentation of $K^{\prime}$, so the theorem is true when $\Delta(\gamma, m)>1$. 
Now assume $\Delta(\gamma, m)=1$. Let $\alpha \cup \beta$ be a 1-tunnel presentation of $K$, and let $W=$ $M-\operatorname{Int} N(\beta)$. By Lemma 3.2, $M^{\prime}=(M, K ; \gamma) \cong \tau(W, c)$ for some $c$ on $\partial W$ which intersects the annulus $A=W \cap N(\beta)$ in a single arc. From the proof, we know that $K^{\prime}$ is isotopic to the central curve $s$ of $A$, which can be written as $\bar{\alpha} \cup \bar{\beta}$, where $\bar{\beta}=s \cap N(c)$, and $\bar{\alpha}=s-\operatorname{Int} \bar{\beta}$. Note that $\bar{\beta}$ is a properly embedded arc in $\tau(W, c)$, and $\bar{\alpha}$ lies on the boundary of $\tau(W, c)=M^{\prime}$. We want to show that it is a 1-tunnel presentation of $K^{\prime}$, i.e., there is a compressing disk of $\partial M^{\prime}$ which is disjoint from $\bar{\beta}$.

There is a compressing disk of $\partial M$ disjoint from $\beta$, which remains a compressing disk of $\partial W$ in $W$, so $\partial W$ is compressible. Since $M^{\prime}=\tau(W, c)$ is also $\partial$-reducible, by the Handle Addition Theorem $\partial W-c$ is compressible. Actually, from the proof in [9], a stronger result is true: There is a compressing disk $D^{\prime}$ of $\partial(\tau(W, c))$ which is disjoint from the attached 2-handle. Since $\bar{\beta}$ lies on the boundary of the attached 2-handle, $D^{\prime}$ is disjoint from $\bar{\beta}$. By definition, $\bar{\alpha} \cup \bar{\beta}$ is a 1-tunnel presentation of $K^{\prime}$.

As a corollary of the results in this section, we have the following duality property of 1-bridge knots in handlebodies.

Theorem 5 Suppose $K$ is a 1-bridge knot in a handlebody $M$ such that some nontrivial surgery on $K$ produces a handlebody $M^{\prime}$. Then the dual knot $K^{\prime}$ in $M^{\prime}$ is also a 1-bridge knot.

Proof. By Theorem $4, K^{\prime}$ is a 1-tunnel knot. Since it is also a $\partial$-reducing knot, by Theorem 3 it is a 1-bridge knot.

We remark that Theorem 3 is not true for general 3-manifold. There are 1-tunnel $\partial$-reducing knot $K$ in some manifold such that neither $K$ nor its dual is 1-bridge. The following is a simple example.

Let $Y$ be the boundary sum of 3 manifolds $X_{1}, X_{2}, X_{3}$, each of which is a (torus $\times I)$. Let $C_{1}, C_{2}$ be curves on $\partial Y$ as shown in Figure 3. Let $M=\tau\left(Y, C_{2}\right)$, and let $K$ be $C_{1}$ pushing into the interior. It can be shown that $\partial M$ is compressible in $M$ and incompressible in $M-K . K$ is clearly 1-tunnel, but it is not 1-bridge because it is not homotopic to a curve on $\partial M$. Using Lemma 3.2, it can be shown that for some $\gamma \neq m$, $(M, K ; \gamma) \cong \tau\left(Y, C_{1}\right)$, and the dual knot $K^{\prime}$ is $C_{2}$ pushing into $M^{\prime}$. This gives the required $\partial$-reducing surgery. We omit the details. 
(Figure 3)

Besides Conjecture A and B, there are some other things about $\partial$-reducing surgeries that are still unknown. The following are two problems.

Problem (1) If $K$ is a 1-tunnel knot but is not 1-bridge, can it admit 2 nontrivial $\partial$ reducing surgeries?

(2) Are there any $\partial$-reducing knots in an anannulus manifold which are not 1-tunnel?

\section{References}

[1] J. Berge: The knots in $D^{2} \times S^{1}$ with nontrivial Dehn surgery yielding $D^{2} \times S^{1}$, preprint.

[2] J. Berge: Knots in handlebodies which can be surgered to produce handlebodies, preprint.

[3] M. Culler, C. Gordon, J. Luecke, and P. Shalen: Dehn surgery on knots, Annals of Math., 125 (1987), 237-300.

[4] D. Gabai: On 1-bridge braids in solid tori, Topology 28 (1989) 1-6.

[5] D. Gabai: 1-bridge braids in solid tori, Topology and its Appl. 37 (1990) 221-235.

[6] C. Gordon: Dehn surgery and satellite knots, Trans. Amer. Math. Soc. 275 (1983) 687-708.

[7] C. Gordon and J. Luecke: Knots are determined by their complements, J. Amer. Math. Soc. 2 (1989) 371-415.

[8] J. Hemple: 3-manifolds, Ann. of Math. Studies 86 (1976), Princeton Univ. Press, Princeton, N.J.

[9] W. Jaco: Adding a 2-handle to 3-manifolds: An application to Property R, Proc. Amer. Math. Soc. 92 (1984) 288-292.

[10] M. Scharlemann: Producing reducible 3-manifolds by surgery on a knot, Topology 29 (1990) $481-500$.

[11] Y-Q. Wu: Cyclic surgery and satellite knots, Topology and its Appl. 36 (1990) 205-208.

[12] Y-Q. Wu: Incompressibility of surfaces in surgered 3-manifolds, Topology (to appear).

\footnotetext{
Department of Mathematics

University of California

Santa Barbara, CA 93106

Current address:

Department of Mathematics

University of Texas at Austin

Austin, TX 78712
} 